\title{
LA INIMPUTABILIDAD POR TRASTORNO MENTAL EN EL CÓDIGO ORGÁNICO INTEGRAL PENAL
}

Leslie Fernanda Santillán Montenegro ${ }^{1^{*}}$, Hugo Bayardo Santacruz Cruz ${ }^{2}$

Abogada por la Pontificia Universidad Católica del Ecuador Sede Ibarra.

${ }^{2}$ Docente de la Pontificia Universidad Católica del Ecuador Sede Ibarra, Escuela de Jurisprudencia.

* Autor para correspondencia: Ifsantillan@pucesi.edu.ec

Recibido: 2019/09/23

D0I: https://doi.org/10.26621/XVI23.2020.12.A05.PUCESI.2550.6684

Aprobado: 2020/11/07

\section{RESUMEN}

El trastorno mental, a través de la historia, ha sido considerado como un punto de desvalor para quien lo padece y más aún si la persona cometía un delito. En tiempos pasados, el enfermo mental era separado de quienes eran considerados sanos, es decir, existía una ruptura entre normales y anormales; actualmente, con el nuevo paradigma del derecho penal, se ha optado por tratar a estas personas acorde a su condición y capacidad, brindándoles garantías específicas.

Este trabajo da cuenta del análisis documental exploratorio que se realizó a diferentes posturas conceptuales referentes al trastorno mental en el ámbito del derecho penal; también se hace alusión a ciertas providencias con las cuales se pone de manifiesto los rasgos tan particulares de este tipo de delitos cometidos por personas que sufren de algún trastorno mental.

Palabras clave: trastorno mental, crimen, sanción penal

\section{ABSTRACT}

Mental disorder, throughout history, It has been considered a point of worthlessness for those who undergo even though that who committed a misdeed. In the past, the mentally ill, was separated from those who were considered healthy, there was a rift between normal and not normal people, currently with the new paradigm of criminal law it has been opt for treating to these people according to their condition and capacity, providing them specific guarantees. This analysis documental research was carried out to different conceptual positions regarding to mental disorder in the field of criminal law, it also refers to certain measures with particular features of this type of crimes committed by people suffering a mental disorder.

Keywords: mental disorder, crime, criminal punishment 


\section{INTRODUCCIÓN}

La presente investigación pretende mostrar la complejidad que para los operadores jurídicos representa el abordaje de la inimputabilidad por trastorno mental, dado que, dentro de la teoría del delito, al analizar la imputabilidad como un elemento de la culpabilidad únicamente se la explica de modo genérico. Es común encontrar en los manuales de derecho penal que la imputabilidad de una persona desaparece cuando esta no alcanza a comprender la ilicitud de su conducta o carece de la capacidad para actuar conforme a dicha comprensión. De esta manera, el fundamento de la exclusión de la responsabilidad penal se lo relaciona directamente con la anormalidad motivacional de la persona (Mir Puig, 2016), dado que la motivación de la norma penal solo puede ser eficaz cuando el individuo "tiene conciencia de la prohibición pues, de lo contrario, éste no tendría motivos para abstenerse de hacer lo que hizo" (Muñoz y Arán, 2010, p. 381).

No obstante, esta explicación no permite que se aborde con detalle el punto central de la inimputabilidad por trastorno mental, que no es más que conocer cuáles son los efectos propios que entrañan las patologías psíquicas y en qué medida esos efectos inciden sobre el sujeto en lo que atañe a la capacidad de comprensión de la ilicitud de su conducta y por ende su responsabilidad penal (Mir Puig, 2016). Surge, así, el importante rol de los peritos médicos legistas, es decir, de aquellos profesionales que poseen conocimientos especiales sobre aspectos de naturaleza biológica o médica, y cuyo aporte en la actualidad es esencial en temas de inimputabilidad, con razón de que estos desde un punto de vista empírico intentan ilustrar y asesorar al jueces o tribunales para que puedan tomar su decisión respecto de un caso concreto.

De este modo, en el análisis de la imputabilidad se presenta una relación muy estrecha entre los operadores jurídicos y los médicos, donde, si bien es cierto que el peritaje psiquiátrico representa un insumo esencial, no es menos cierto que la última palabra la tienen los juzgadores que (mediante un análisis jurídico-normativo) determinarán la imputabilidad 0 no de un sujeto.

En este contexto, el objetivo de esta investigación es mostrar que la alegación de inimputabilidad por trastorno mental (y el consecuente informe pericial que lo confirme) no son indicios suficientes para que el juez 0 tribunal penal determine la exclusión de la culpabilidad de un sujeto, ya que lo verdaderamente determinante es demostrar que al momento de cometer la infracción el sujeto era incapaz de comprender la antijuridicidad de su conducta o de determinarse conforme a ella.

\section{MATERIALES Y MÉTODOS}

La presente investigación es de tipo documental bibliográfico, con un enfoque cualitativo y descriptivo, debido a que la dirección principal del trabajo de investigación recae en recolectar fuentes documentales que sirvan para mostrar un estudio dogmático de la categoría de la culpabilidad y relacionarla con las principales patologías que la psiquiatría contemporánea considera que suprimen la conciencia del sujeto que la padece. Es decir, buscar información sobre ciertas enfermedades mentales que la doctrina mayoritaria ha usado y ha aceptado como causa de inimputabilidad, recordando que dentro de nuestro marco jurídico no existe una lista determinada de enfermedades mentales aceptadas como tal causa, lo cual dificulta a los administradores de justicia actuar con discernimiento sobre estos casos.
Adicionalmente, se analizó la norma penal que excluye la culpabilidad y la aplicabilidad que tienen dentro del proceso penal; para ello, se recurrió al estudio de los Proceso N. 10281-2017-00838 y N. 10281-2017-00638, utilizando el estudio de caso como metodología y se identificó la valoración que los operadores jurídicos tienen respecto de las personas que padecen algún tipo de trastorno mental al momento de cometer un delito.

\section{RESULTADOS Y DISCUSIÓN}

\section{La salud mental de la población ecuatoriana}

La Organización Mundial de la Salud (OMS) describe a la salud mental como "un estado de bienestar en el cual el individuo se da cuenta de: sus propias aptitudes, puede afrontar las presiones normales de la vida, puede trabajar productiva y fructíferamente y es capaz de hacer una contribución a su comunidad" (Petra, 2002, p. 1).

Bajo la denominación de enfermedades mentales, se agrupan una amplia tipología de trastornos que afectan, con carácter temporal 0 permanente, las diversas facultades psíquicas del ser humano. Es de señalar que la psiquiatría moderna ha abandonado en buena parte la clasificación morfológica de la psiquiatría tradicional. En la actualidad los modelos de clasificación de las enfermedades psíquicas y variantes más utilizados son: 1) la Clasificación Internacional de Enfermedades (CIE-10) de la OMS, que cambiará a partir del 1 de enero de 2022 a la versión CIE-11; y, 2) el Manual Diagnóstico y Estadístico de los Trastornos Mentales (DSM-5) de la American Psychiatric Association, del cual toma la OMS su clasificación (Calabuig \& Villanueva Cañadas, 2004, p. 1059).

Por su parte, la Organización Panamericana de la Salud (PAHO), sobre la variedad de patologías psíquicas existentes, señala que la depresión y la ansiedad son los trastornos mentales más prevalentes en la población, pues calcula que a nivel mundial $4,4 \%$ de la población sufre un trastorno depresivo y 3,6\%, un trastorno de ansiedad (Organización Panamericana de la Salud, 2017).

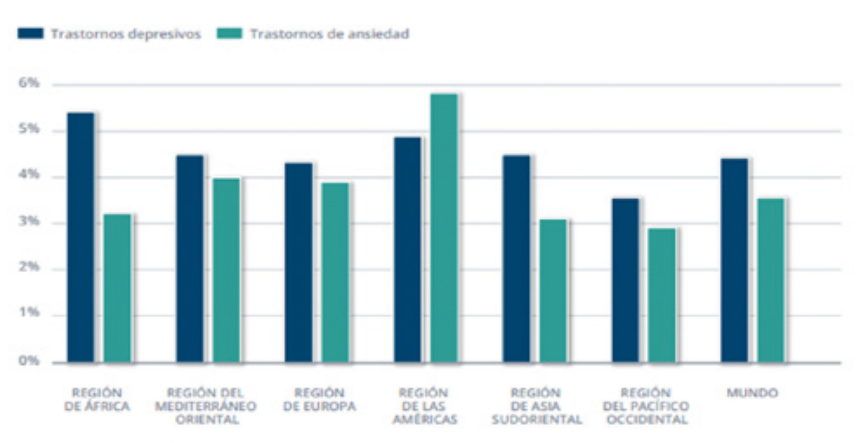

Figura 1. Prevalencia de trastornos mentales comunes (porcentaje de la población), por Región de la IMS

Fuente: Organización Panamericana de la salud, 2017

En el caso de Ecuador, la prevalencia de trastornos mentales comunes se encuentra por encima de la media de la Región de las Américas. De acuerdo a esta organización, el 8,3\% de la población ecuatoriana padece depresión, mientras que el 5,2\% sufre de ansiedad (Organización Panamericana de la Salud , 2017). En América Latina (incluyendo Ecuador) es común la presencia de personas que sufren trastornos mentales graves en las calles de las distintas ciudades del país. Su aspecto peculiar ha tejido sobre ellas un estigma, dado que estos representan algo que no es habitual en la generalidad de las personas 0, por mejor decir, de la sociedad (Mascayano, 2015). 
En este sentido, es de resaltar que, ligado al cometimiento de crímenes atroces, generalmente se encuentra aparejado en el imaginario social la actuación de una persona que padece algún tipo de trastorno mental grave, cosa que no es del todo cierta, pues desde los iniciales estudios se ha establecido que "la delincuencia entre los enfermos mentales, globalmente considerados, no constituye sino una pequeña parte de la criminalidad en general” (Calabuig \& Villanueva Cañadas, 2004, p. 1040).

\section{Trastornos mentales indiciarios de inimputabilidad}

Introduciendo el autor Frank (2002), este señala que la culpabilidad debe enmarcarse en 3 elementos: "1.) aptitud normal del autor; 2) una cierta concreta relación psíquica del autor con el hecho en cuestión 0 al menos la posibilidad de estas (dolo 0 imprudencia) y 3) normalidad de las circunstancias en las que actúa el sujeto" (Frank, 2002), para ser considerado como responsable penal. Complementando lo anterior, Goldschmidt (2002) dice que el sujeto que comete el delito -para que sea considerado como responsable- debió haber tenido una motivación normal y conocer que su conducta iba a resultar en un daño, por lo que si este no es consciente de esta violación no es responsable de lo cometido (Goldschmindt, 2002).

Tabla 1. Diferencia: Culpabilidad entre sujetos normales y de aquellos que padecen de un trastorno mental

\begin{tabular}{lll}
\hline $\begin{array}{c}\text { Culpabilidad/ } \\
\text { preceptos }\end{array}$ & \multicolumn{1}{c}{ Sujeto normal } & $\begin{array}{c}\text { Sujeto con trastorno } \\
\text { mental }\end{array}$ \\
\hline & $\begin{array}{l}\text { El sujeto tiene la capaci- } \\
\text { dad mental de conocer }\end{array}$ & $\begin{array}{l}\text { En el momento de come- } \\
\text { ter la infracción el sujeto }\end{array}$ \\
1.Capacidad & lo ilícito de su actuar, & padece de una alteración \\
de culpabili- & y sabiéndolo comete & psíquica grave, por ende, \\
dad & $\begin{array}{l}\text { la conducta que va en } \\
\text { no comprende lo que } \\
\text { contra de la norma. }\end{array}$ & hizo.
\end{tabular}

\begin{tabular}{lll}
\hline & $\begin{array}{l}\text { Es imputable, el sujeto } \\
\text { posee una comprensión }\end{array}$ & Es inimputable, tiene in- \\
& no necesariamente téc- & prender el mal de su \\
2.Conocimien- & nica de lo contrario a la & actuar, hay un desapego \\
to de antijuri- & norma, y la conciencia & con la realidad. \\
cidad & o facultad de palpar la \\
& realidad del acto ilícito \\
& de su actuar. & \\
\hline \multirow{3}{*}{ 3.Exigibilidad } & $\begin{array}{l}\text { El sujeto puede y debe } \\
\text { responder por su ilícito, ducta es inexigible } \\
\text { ya que actuó desafian- } \\
\text { do a la norma }\end{array}$ \\
\hline
\end{tabular}

En el Ecuador no existe un catálogo de trastornos mentales aceptados como causa de inimputabilidad. Sin embargo, es comúnmente aceptada por jueces y tribunales las referencias de las perturbaciones o alteraciones mentales formuladas por la doctrina mayoritaria.

\section{a) Las psicosis}

La psicosis ocurre cuando una persona pierde contacto con la realidad es una condición mental grave; se caracteriza por la incapacidad de cuidar de sí mismo y la posibilidad de lesionar a otros (Vega Gutiérrez, 2005).

Según la OMS, (2019) este trastorno afecta alrededor de 21 millones de personas de todo el mundo; dentro de este grupo, los trastornos más comunes son la esquizofrenia y la paranoia. Un punto interesante es que este trastorno puede ser producido por abuso de sustancias alcohólicas y drogas o por una profunda depresión y son las causas de inimputabilidad por excelencia (Vega Gutiérrez, 2005).

\section{b) Las oligofrenias}

También conocidos como trastornos de desarrollo psicológico, abarcan la discapacidad intelectual causando una disfunción en las habilidades cognitivas y en la conducta (Organización Mundial de la Salud, 2019). En cuanto a esta anomalía psíquica, se señala que no son verdaderas alteraciones de la mente; por lo tanto, el juez debe hacer una valoración específica en cuanto a la intensidad del trastorno, ya que solo se pueden considerar como causa de inimputabilidad aquellos trastornos graves que afecten en la totalidad de la comprensión del injusto penal (Vega Gutiérrez, 2005).

\section{c) Las psicopatías}

Este trastorno es considerado como las "anormalidades que inciden en la personalidad del sujeto, se caracteriza por un desequilibrio cuantitativo de los distintos componentes de la personalidad" (Vega Gutiérrez, 2005, pág. 8); constituyen los trastornos de personalidad e indican un alto grado de peligrosidad criminal para quien la padece, ya que pueden ser capaces de cometer delitos inhumanos (ibíd.)

\section{d) Las neurosis}

Se las define como las "reacciones psíquicas anómalas, ante determinadas situaciones, que se manifiestan principalmente en forma de angustia, depresión o mecanismos de defensa contra ambos, pero que pueden llegar a generar impulsos irreversibles" (Vega Gutiérrez, 2005).

\section{La valoración de la inimputabilidad por trastorno mental a la luz de la jurisprudencia}

Primeramente, es preciso aclarar que el derecho penal también rige a las personas que no están dentro de una situación psíquica normal; por ejemplo, aquellos que padecen de un trastorno mental.

Es así que el término trastorno mental, desde el punto de vista jurídico penal ecuatoriano, hace mención a:

La persona que al momento de cometer la infracción no tiene la capacidad de comprender la ilicitud de su conducta 0 de determinarse de conformidad con esta comprensión, en razón del padecimiento de un trastorno mental, no será penalmente responsable. En estos casos la o el juzgador dictará una medida de seguridad. (art. 36 Código Orgánico Integral Penal, 2014)

Del artículo manifestado se desglosan tres presupuestos importantes: a) Momento de cometer la infracción: para que se dé este presupuesto es vital que la perturbación 0 alteración mental esté presente en el preciso momento de cometer el delito; según Roxin (vid. Vega Gutiérrez, 2005) en este punto es fundamental la participación de un médico perito quien debe determinar de acuerdo a su experticia si el sujeto era asequible a la norma en el momento del hecho.

b) Incapacidad de comprender la ilicitud: este presupuesto va de la mano de la inimputabilidad, ya que esta figura cubre la incapacidad del autor de entender el mal de una conducta regulada por el derecho penal; esta incapacidad no le "permite al sujeto percatarse de que está lesionando o poniendo en riesgo 
a un bien jurídico típicamente tutelado" (Gaviria Trespalacios, 2005, p. 34), lo que le inhabilita para comportarse de manera jurídicamente aceptable.

Acotando a lo expuesto, el tratadista Terragni "estable que la inimputabilidad es aquella que indica ciertos estados mentales que son causantes principales de la imposibilidad de comprender la criminalidad del acto, estas causas son indicadas por la ley". (Terragni, 1981); así también, Roxin "establece que el inimputable es aquel que no puede hacer nada al respecto" (Roxin, 1981, ) en cuanto al cometimiento del delito, ya que su actuar en ese momento no está vinculado con la realidad y no sabe la maldad de su acto.

c) Ausencia de responsabilidad penal: "serán todas aquellas que niegan alguna de las condiciones imprescindibles para que exista responsabilidad penal" (Fernández Sandoval, 2003), es decir, una conducta típica, antijurídica y culpable. En el art 35, el COIP establece que una causa de inculpabilidad es el trastorno mental debidamente comprobado; por tal motivo, quien lo padezca no será responsable penalmente, ya que no se cumple con todas las categorías dogmáticas del delito.

Dentro del art 36 Código Orgánico Integral Penal (COIP), en su apartado segundo, se informa sobre la semi-imputabilidad y la doctrina penal lo entiende como imputabilidad disminuida, ya que el sujeto comete el ilícito con disminución en su capacidad volitiva y cognoscitiva, es decir, que en cierta medida la persona tiene conocimiento de lo que está haciendo, encontrándose, no obstante, en un estado de anormalidad psíquica pero no de total incapacidad. (Cueva Tamariz, 2004)

\section{Tratamiento penal para los inimputables. Medidas de seguridad}

Una vez indicados los aspectos más trascendentes en cuanto al trastorno mental en la normativa penal ecuatoriana, cabe mencionar que a la persona que padece de un trastorno mental debidamente comprobado por un especialista en el tema (perito psiquiatra) no se le puede penar de la misma forma que a una persona normal, y al tener un Derecho Penal dualista conformado por penas y medidas, lo correcto para su sanción es usar una medida de seguridad, ya que esta va acorde a su condición.

Dentro de la doctrina, "la medida de seguridad es una consecuencia jurídica aplicada a una persona física en función de la peligrosidad de su hecho. No se imponen en función de la culpabilidad, pues es precisamente ésta la que les falta para responder penalmente" (Fernández, Vallejo, \& Perrino Pérez, 2017).

Continuando con este concepto, Diez Ripollés (vid. Núñez Fernández, 2009) señala que la peligrosidad dentro de un ámbito jurídico penal es entendida como la probabilidad alta de que el sujeto vuelva a delinquir, más aún si este padece de algún trastorno mental y ahí recae la importancia de una medida de seguridad bien determinada en favor de la naturaleza del delito y su gravedad, cuyo fin será evitar o prevenir la reincidencia.
Tabla 2. Diferencias: penas y medidas

Diferencia entre penas y medidas

Pena

Medida

1. Se encarga de velar por la pre- 1. Está enfocada en asegurar la vención general y especial. prevención especial

2. Hay un reproche moral que 2. No hay reproche realiza la sociedad

3. Tiene como fin la protección

3. Tiene como fin la restauración de la sociedad y rehabilitación del del orden jurídico individuo sujeta a esta

4. Va dirigida a todo sujeto que 4. Va dirigida a ciertos sujetos por sabe el mal de su actuar y aun sus condiciones de no entender así lo hace la ilicitud de su acto

Fuente: Sánchez, R. W.

- Procedimiento para el conocimiento de delitos cometidos por personas con trastornos mentales

El Código Orgánico Integral Penal (2014) tiene como una de sus finalidades establecer el procedimiento para el juzgamiento de las personas con estricta observancia del debido proceso; por tal motivo, en su art 588 establece que:

"Si la persona investigada o procesada muestra síntomas de trastorno mental, la o el fiscal ordenará su inmediato reconocimiento, para cuyo fin designará a un perito médico psiquiatra, quien presentará su informe en un plazo determinado. De este informe dependerá el inicio de la instrucción, la continuación del proceso o la adopción de medidas de seguridad, según el caso".

Para un adecuado y oportuno procedimiento se debe hacer referencia a la Resolución No. CJ-DG-2015-10, en la cual se manifiesta la guía para el conocimiento de los delitos cometidos por las personas con trastornos mentales.

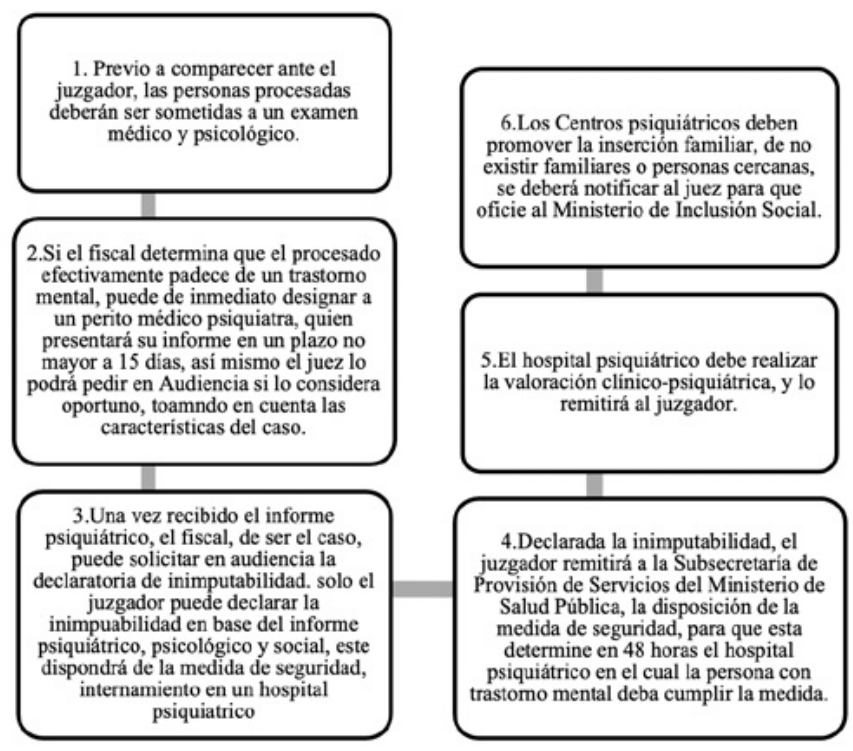

Figura 1. Procedimiento para delitos con indicios de trastorno mental Fuente: Resolución No. CJ-DG-2015-10

- Estudio de casos en normativa: Código Orgánico Integral PenalFiscalía de Imbabura- Personas y Garantías N.2 


\section{PRIMER CASO}

Proceso N. 10281-2017-00638

Asunto: Asesinato (art. 140)

\section{ANTECEDENTES}

El día 19 de abril del 2017, en el domicilio de Jaime Benítez, miembros policiales acuden a su domicilio; al ingresar, pudieron observar al ciudadano Emerson Benítez, quien les manifestó que había tenido una discusión con su hermano (de nombre Jaime Benítez) y que producto de ésta, su hermano se había suicidado y se encontraba en el segundo piso. Sin embargo, al ir observando el inmueble, en el inicio de las gradas que conducen a la segunda planta había gran cantidad de manchas y salpicaduras de color rojo; una vez en la segunda planta, se observó en la puerta de ingreso del dormitorio a un cuerpo de sexo masculino en posición decúbito dorsal, el cual era atendido por un paramédico del cuerpo de Bomberos, quien había indicado que (a simple vista) el cuerpo presentaba varias heridas corto-punzantes en el rostro, espalda y cuello; se toma procedimiento y se procede a llamar a la señora Fiscal de turno, quien luego de conocer los hechos ha dispuesto la aprehensión del ciudadano Emerson Benítez.

\section{Actuaciones judiciales:}

1. Valoración psicológica forense: el sujeto pasivo padece de un Trastorno Delirante; recordando lo expuesto por la doctrina mayoritaria, este trastorno causa inimputabilidad, ya que se encuentra dentro de la psicosis y este causa una pérdida total con la realidad que lo rodea, por lo que su actuar es violento e indescifrable (Ortuño \& Gutiérrez-Roja, 2011).

2. Examen psiquiátrico actual: pensamientos con ideas delirantes de daño y perjuicio, juicio, raciocinio y voluntad afectadas en forma parcial, el examinado presenta una afectación mental grave, combinada con que -con mucha probabilidad- al momento en que ocurrieron los hechos también se encontraba en consumo de drogas, lo cual le afectó la conciencia y la voluntad en forma completa. De lo expuesto, es claro manifestar que el sujeto al momento de cometer el delito no tuvo percepción de su actuar, es decir, no entendía lo que estaba haciendo, esto es, el sujeto no conocía el daño que estaba realizando a la otra persona.

3. Decisión: el juez declara como inimputable al señor Emerson Benítez, y dicta la correspondiente Medida de Seguridad a ejecutarse en un hospital psiquiátrico.

En este caso, se puede observar un correcto procedimiento para tratar delitos con indicios de trastorno mental: para comenzar hay un total discernimiento del fiscal sobre la conducta del sujeto pasivo. Es preciso recordar que, si el fiscal tiene presentimiento de que el sujeto pasivo muestra una conducta irracional o extraña, puede pedir de forma inmediata la valoración psicológica y el examen psiquiátrico adecuándose al debido proceso; ello significa que dentro del proceso mencionado el fiscal actúa conforme lo emana la norma.

A través del correspondiente examen psiquiátrico, se logra visualizar el cumplimiento de los presupuestos necesarios para incurrir en un caso de trastorno mental, ya que el sujeto pasivo, al momento de cometer el delito, está afectado en forma total sobre su capacidad de comprender la ilicitud de lo que estaba realizando, ergo había un total desplazamiento de la realidad. Al finalizar el juez acierta en imponerle una medida de seguridad, ya que esta se adecua a su condición, buscando la correcta rehabilitación del sujeto y sobre todo percatándose de poner un límite a la conducta del mismo mediante ayuda especializada, en este caso el internamiento en un hospital psiquiátrico.

\section{SEGUNDO CASO:}

Proceso N. 10281-2017-00838

Asunto: Incumplimiento de decisiones legítimas de autoridad competente (art. 282, inc. 1).

\section{ANTECEDENTES}

El día 24 de mayo del 2017, la policía se trasladó hasta el domicilio de la señora María A., quien les ha autorizado el ingreso a su domicilio y también les presentó una boleta de auxilio, en contra de la señorita Karla R. -su hija-, misma que en el transcurso del día le había agredido verbalmente y psicológicamente, con palabras como: "que quiero matarte, quiero que desaparezcas para quedarme con las cosas, quiero quemar la casa, tengo unos amigos que ellos saben cómo hacer las cosas, eres una longa chiquita", por lo que la víctima ha manifestado que quiere hacer uso de la boleta de auxilio, ya que no es la primera vez que suceden estos inconvenientes, razón por la cual Karla ha sido aprehendida.

\section{Actuaciones judiciales:}

1. Llamamiento a juicio: En lo principal se dijo: que la señorita Karla actuó con conocimiento y voluntad en todo y por ende se la penó, como autora del delito de Incumplimiento.

2. Tribunal Único de garantías Penales: declaran la nulidad de lo actuado, por la ausencia de un diagnóstico definitivo sobre la evaluación psiquiátrica que determine la inimputabilidad; también se indica que se tome en cuenta que fue agredida e intentaron abusar sexualmente de ella en la cárcel de mujeres.

3. Decisión: el juez, a través del informe psiquiátrico, concluye que la ciudadana Karla R. presenta una enfermedad mental orgánica grave que le afecta la conciencia y voluntad llamado "Trastorno metal: borderline (psicopatía)", y se resuelve declararla inimputable disponiendo medida de seguridad; el juez llega a dar esta decisión debido a que, una vez analizado el examen psiquiátrico, se determina que Karla no podía controlar sus acciones, debido a que la característica de este trastorno es la anormalidad de la personalidad.

En este caso, las actuaciones judiciales no son las más idóneas, ya que se hace caso omiso de que el sujeto pasivo sufría de ciertas anomalías en su comportamiento desde niña, lo cual era un punto de alerta. Tampoco se presta atención a las características tan particulares del caso, ya que dentro del informe policial, se establece la conducta errática y explosiva del sujeto pasivo, lo cual demostraba una conducta anormal. La norma es precisa en señalar que, si el fiscal no pudo discernir esta conducta, el juez al ser dador de derecho tiene la obligación de observar las circunstancias específicas del caso y así determinar lo más oportuno para tratarlo.

No obstante, el Tribunal, velando por la seguridad jurídica de las partes procesales, declara la nulidad de lo actuado en la unidad, al no respetar el debido proceso, incumpliendo con la diligencia debida de pedir el informe psicológico, psiquiátrico y social, cuando es aparente que la persona procesada muestra síntomas de trastorno mental.

Para este tiempo que pide la nulidad, la procesada ya se encontraba privada de su libertad en la cárcel de mujeres de Tulcán; en este sitio fue agredida físicamente e intentaron abusar de ella, ya que las reclusas no entendían su condición psíquica. Es claro que cuando no se respeta lo dictado por la norma se llega a vulnerar derechos. 
A pesar del tiempo transcurrido, la administración de justicia logró determinar que el sujeto pasivo sufría de un trastorno mental y el juez pudo por tanto dictar medida de seguridad con el fin de tratar esta anomalía en su conducta.

\section{CONCLUSIONES}

Para entender al trastorno mental hay que hacer alusión a la historia humana, ya que, conforme pasa el tiempo, se ha ido entendiendo al que padece de un trastorno mental con acepciones diferentes; en un principio eran llamados locos y simplemente eran excluidos de la sociedad. Actualmente, forman parte de un grupo vulnerable, con derechos y garantías específicos, según lo determina la Constitución del Ecuador. Dentro de la normativa penal, es un tema delicado, no obstante, el legislador ha tipificado al trastorno mental al tenor de 3 presupuestos esenciales: a) momento de cometer la infracción, b) incapacidad de comprender la ilicitud de su conducta y c) ausencia de responsabilidad penal; con estos se pretende respetar el debido proceso y el principio de igualdad dentro de las actuaciones judiciales, con el fin de no vulnerar derechos y actuar con diligencia dentro de los procesos.

Así, cuando se entiende que la persona con trastorno mental carece de voluntad y conocimiento de su actuar y por ende, no comprende la pena, merece un trato específico si ha cometido un delito, ya que su situación no es igual a la de una persona normal; a lo expuesto el COIP recoge la medida de seguridad de internamiento en un hospital psiquiátrico, lo cual garantiza la protección de estas personas y sobre todo previene un acto pos delictivo que ponga en riesgo a la sociedad. Cabe señalar que el fin de la medida es resguardar la seguridad de la sociedad y también ayudar a rehabilitarse a la persona que tenga el padecimiento mental.

La única figura judicial que puede declarar la inimputabilidad es el juez; en este sentido, es necesario recalcar que él necesita que todas las actuaciones judiciales estén enmarcadas en el debido proceso, porque de lo contrario podría dictarse una decisión errónea vulnerando derechos y transgrediendo; para evitar una decisión inadecuada, por tanto, resulta vital contar con el informe psiquiátrico, psicológico y social.

El papel del fiscal en la etapa pre procesal de este tipo de delitos es de suma importancia, ya que este es el primer filtro para discernir si la persona que cometió el delito padece de una enfermedad mental y este discernimiento se logra con experiencia laboral y adecuando una correcta entrevista al procesado. Parece obvio que sería de gran ayuda tener una lista determinada de las enfermedades mentales que sean causas de inimputabilidad, teniendo ello como fin que el operador de justicia enmarque su actuar o decisión sobre ciertos lineamientos, a fin de no caer en alguna actuación inoportuna.

En el Ecuador, no existe un catálogo numerus clausus que determine cuáles son los trastornos mentales aceptados dentro del ámbito penal para ser considerados como causas de inimputabilidad; por lo tanto, entra a ser una norma penal en blanco, lo que implica que haya la necesidad de referirse a ciertas normas internacionales o la doctrina mayoritaria para definirlos, que consideran a la psicosis, oligofrenias, psicopatías y neurosis como el grupo de trastornos mentales aceptados como causas de inimputabilidad.

\section{REFERENCIAS BIBLIOGRÁFICAS}

Calabuig, G., \& Villanueva C, E. (2004). Medicina legal y toxicología. Recuperado de https://archive.org/stream/Forense.Forensic. Medicina.Legal.Toxicologia.Gisbert.Calabuig.Villanueva.Canadas.SPAIN.66.Edicion/Forense.Forensic.Medicina.Legal.Toxicologia.Gisbert.Calabuig.Villanueva.Ca\%C3\%B1adas.SPAIN.6\%C2\%AA.Edicion_djvu.txt

Cueva T, A. (2004). Introducción a la psiquiatría forense. Cuenca: Universidad del Azuay.

Fernández S, J. (2003). Causales de ausencia de responsabilidad. Dia/net. Recuperado de https://dialnet.unirioja.es/servlet/articulo?codigo $=2347527$

Fernández, E., Vallejo, M., \& Perrino Pérez, M. (2017). Penas, medidas y otras consecuencias jurídicas del delito (1.a ed.). Dykinson, S.L. https://doi.org/10.2307/j.ctt1zgwjd8

Frank, R. (2002). Sobre la Estructura del Concepto de Culpabilidad. Buenos Aires: $B$ de $f$.

Gaviria T, J. (2005). La inimputabilidad: concepto y alcance en el código penal colombiano. Redalyc.org. Recuperado de https://www. redalyc.org/articulo.oa?id=80617859005

Goldschmindt, J. (2002). La concepción normativa de la culpabilidad (2.a ed.). B de f. Recuperado de http://www.poderjudicial. gob.do/consultas/biblioteca/Textos/LA\%20CONCEPCION\%20 NORMATIVA\%20DE\%20LA\%20CULPABILIDAD\%20-\%20 James\%20Goldshmidt.pdf

Mascayano, F. (2015). Estigma hacia los trastornos mentales: características e intervenciones. Scielo. Recuperado de scielo.org. $\mathrm{mx}$ : http://www.scielo.org.mx/scielo.php?script=sci_arttext\&pi$d=S 0185-33252015000100008$

Mir Puig, S. (2016). Derecho penal parte general (10 ed.). Barcelona, España: EDISOFER S.L.

Muñoz, F. y García, M. (2010). Derecho Penal. Parte General. Velencia: Tirant lo Blanch.

Núñez F, J. (2009). La peligrosidad postdelictual del enfermo mental inimputable y su gestión jurídico penal. Recuperado el 2020, de Dialnet: https://dialnet.unirioja.es/servlet/articulo?codigo $=2947704$

Organización Mundial de la Salud. (2019). CIE-11. Clasificación Internacional de Enfermedades, 11.a revisión. Recuperado de icd.who. int: https://icd.who.int/es

Organización Panamericana de la Salud . (2017). Depresión y otros trastornos mentales comunes. Estimaciones sanitarias mundiales. Recuperado de https://iris.paho.org/: https://iris.paho. org/bitstream/handle/10665.2/34006/PAHONMH17005-spa. pdf? sequence $=1$ \&isAllowed $=y$

Ortuño, F., \& Gutiérrez-Roja, L. (2011). Psicosis. Uned. Recuperado de https://www2.uned.es/psicofarmacologia/stahl4Ed/contenidos/ Tema1/documentos/C4/C4D_11.pdf

Petra, I. (2002). Concepto de salud mental y normalidad. Recuperado el 2020, de psiquiatria.facmed.unam: http://psiquiatria.facmed. unam.mx/docs/ism/unidad1.pdf

Roxin, C. (1981). Culpabilidad y Prevención en Derecho Penal. Munich: Reus, s.a.

Sánchez R, R. (2012). "Distinción entre penas y medidas de seguridad en la codificación mexicana y análisis comparativo con el código penal español" (Tesis doctoral) . Recuperado de http://master. us.es/: http://master.us.es/cuadernosmaster/17.pdf

Terragni, M. A. (1981). Culpabilidad penal y responsabilidad civil. Buenos Aires: Hammurabi.

Vega G, Z. (2005). Las alteraciones o perturbaciones psíquicas como 
causas de inculpabilidad: problemática especial en el ámbito de las psicopatías. Revista Encuentrol UCA de Nicaragua. Recuperado de https://www.uca.edu.ni/2/index.php/portada-revista-encuentro

\section{REFERENCIAS LEGALES}

- Constitución de la República del Ecuador [CRE], de 20 de octubre de 2008.

- Código Orgánico Integral Penal [COIP], de 10 de febrero de 2014 (Ecuador). Registro Oficial S. 180. Art. 35,36. 588.

- Resolución N, CJ-DG-2016-10 (Guía para el conocimiento de delitos cometidos por personas con trastornos mentales), de 1 de febrero de 2016.

- Asesinato, 10281-2017-00638 / (Fiscalía de Imbabura- Personas y Garantías N.2 2017)

- Incumplimiento de decisiones legítimas de autoridad competente, 10281-2017-00838 / (Fiscalía de Imbabura- Personas y Garantías N.2 2017) 